\title{
Utilização de um Serviço de Monitoria Virtual Voltado para o Ensino de Epidemiologia na Graduação Médica
}

\author{
ANA LUIZA ALFAYA GALLEGO SOARES \\ CLARISSE PEREIRA DIAS \\ EDISONIGLESIAS DE OLIVEIRA VIDAL \\ CLÁUDIA MEDINA COELI \\ LIZ MARIA DE ALMEIDA ${ }^{5}$ \\ KENNETH ROCHEL DE CAMARGO JÚNIOR \\ RESUMO
}

Este artigo discute o ensino de conteúdos de Saúde Coletiva no curso de graduação de Medicina, com base numa experiência desenvolvida na Faculdade de Medicina da UFRJ, Brasil. A monitoria virtual (MV) funciona como um instrumento complementar de ensino e constitui-se de um serviço de respostas a perguntas de alunos, de um website e de um serviço de maladireta eletrônica. Este artigo mostra os resultados da avaliação da utilização e receptividade dos serviços MV no período entre 2000 e 2002. A metodologia consistiu da análise das mensagens enviadas e da avaliação dos serviços, pelos alunos. Neste período, a MV recebeu 153 mensagens contendo 239 perguntas. Mais de $60 \%$ dos alunos receberam alguma mensagem em sua caixa postal e $10 \%$ tiveram contato com as mensagens através de outro aluno. Menos de 20\% enviaram mensagens para a MV. A análise do número de mensagens enviadas mostra uma variação durante o curso com maior concentração de envio próximo às provas da disciplina (35\%). As mensagens continham "dúvidas acadêmicas" em 59\% dos casos. A MV foi avaliada por $86 \%$ dos alunos, dos quais $61 \%$ ficaram totalmente satisfeitos com as respostas recebidas, $18 \%$ afirmaram que a MV aumentou sua freqüência de uso da Internet e $88 \%$ consideraram uma boa idéia a incorporação do serviço em outras disciplinas. O site foi acessado por $64 \%$ dos alunos. A maioria (65\%) realizou entre três e dez acessos. A metodologia utilizada permitiu realizar uma análise exploratória dos possíveis fatores associados à incorporação da intervenção proposta.

Palavras-chave: Epidemiologia; educação médica; graduação; comunicação mediada por computador; Internet. 


\section{Introdução}

A necessidade de aprimoramento do processo ensino-aprendizagem no campo da medicina vem sendo objetivo de reflexão pelo menos desde fins do século XIX (Swanson et al., 1993), evidenciado pelo número crescente de textos publicados a respeito de aspectos críticos onde mudanças se faziam e se fazem necessárias. É surpreendente reconhecer, já em documentos da década de 30 do século passado, a percepção da necessidade de desenvolvimento de hábitos de estudo e pensamento independente nos estudantes de medicina, a fim de proporcionar a auto-educação para toda vida. Igualmente, reconhecia-se como problemática a ênfase dos cursos médicos na memorização de conteúdos, em contraposição ao desenvolvimento de hábitos mais adequados de estudo e diligência intelectual (Rappleye, 1932).

Desde então, muitos têm sido os eixos de debate do ensino médico, dentre os quais é interessante citar, além daqueles mencionados acima, a questão da integração entre ciclo básico e ciclo profissional, bem como entre atividades práticas e teóricas (Ribeiro, 2002); criação de sistemas de avaliação condizentes com os objetivos de aprendizado estabelecidos (Blue et al., 2000; Swanson et al., 1993; Wilkes, 1999); desenvolvimento de habilidades de manejo da informação médica (Charlile et al., 1998; Koschmann, 1995; WFME, 1998; Swanson et al., 1993); capacidade de trabalhar em equipe (Owston, 1997); formação de profissionais éticos e com bom senso de responsabilidade social (Boelen et al., 1995; Ribeiro, 2002) dentro das perspectivas de custeio dos cursos. Pensamos que estas questões se interrelacionam entre si e que, no mais das vezes, ao abordar determinado aspecto dos cursos médicos, outros pontos vêm à tona.

Este trabalho toma como ponto de partida uma experiência adotada no ensino da Epidemiologia dentro do curso de graduação médica da Universidade Federal do Rio de Janeiro (UFRJ). Os temas da epidemiologia constituem ferramenta essencial para o desenvolvimento das habilidades de manejo da informação médica e, conseqüentemente, do pensamento crítico e da capacidade de estudo auto-orientado para além do período de estudo formal na faculdade. À medida que os alunos vão conquistando a compreensão sobre diferentes metodologias de pesquisa e desenhos de estudo, suas vantagens e limitações, vieses e fatores de confundimento vão também se apoderando de recursos argumentativos que os permitem exercer, de forma mais apurada, a reflexão e o julgamento a respeito das publicações a que têm acesso e sobre os demais mecanismos de construção do conhecimento 
médico. Abre-se uma porta para o que Paulo Freire chamou de "curiosidade epistemológica" (Freire, 1997), referindo-se à curiosidade que abandona a posição ingênua de ausência a toda explicação simplista, que se torna rigorosa e sistemática na avaliação de argumentos e perpetua-se enquanto curiosidade, enquanto prazer de descobrir a realidade.

A disciplina de Epidemiologia, juntamente com os outros componentes do campo da Saúde Pública, insere no currículo médico a reflexão em torno da saúde de uma coletividade de pessoas, dos indicadores a partir dos quais esta é estudada, a partir do qual pode emergir a questão da responsabilidade social da prática médica. Essa questão é mais intensa em países em condições socioeconômicas como a brasileira, que, por vezes, se perde dentro da perspectiva individual, e onde, contraditoriamente ao termo "indivíduo", a categoria é a doença (Camargo Jr., 1997).

Uma linha de argumentação que se repete ao longo da literatura recente a respeito do ensino-aprendizagem médico é a do aumento exponencial do volume de conhecimento médico produzido no mundo (Carlile et al., 1998; Hagdrup et al., 1999; Koschmann, 1995; Owston, 1997; Peixoto et al., 2000; Swanson et al., 1993; WFME, 1998) como um dos fatores imperativos para mudanças curriculares nas escolas médicas, no sentido de tornar os estudantes capazes de lidar de forma ótima com esses recursos, contornando o risco de uma "sobrecarga de informação". A idéia da "sobrecarga de informação" é um conceito interessante (Peixoto et al., 2000; Swanson et al., 1993; WFME, 1998), na medida em que chama a atenção para o risco de transformar "o paraíso da informação no inferno da informação" (MacKenzie, 1998), quando a captação de informação - informação esta que não é disponível de forma organizada e nem sempre confiável - não é acompanhada pelo desenvolvimento de julgamento crítico adequado.

Esta lista de argumentos toma um rumo já consensual a respeito da importância de os estudantes dominarem o arsenal de recursos computacionais da denominada "tecnologia da informação" para lidarem com o mundo de dados disponíveis (Abrasco, 2000; Carlile et al., 1998; Koschmann, 1995; Swanson et al., 1993; WFME, 1998). Todavia ressalta-se, com propriedade, a importância de se compreender tais tecnologias não como um objetivo em si mesmas, mas sempre como um recurso a servir aos interesses e objetivos educativos estabelecidos (Koschmann, 1995; WFME, 1998). O essencial não é o uso do computador ou da Internet pura e simplesmente, mas a forma como este uso é conduzido dentro do contexto do ensino-aprendizado (Owston, 1997).

A partir destas perspectivas, vem sendo desenvolvido desde 2000, no 
curso de Epidemiologia para graduação médica da UFRJ, a atividade denominada Monitoria Virtual em Epidemiologia. A monitoria virtual funciona como um instrumento complementar de ensino ao curso. A disciplina de Epidemiologia ocorre no quinto período letivo dos estudantes, em meio à transição do ciclo básico para o profissional, quando se intensificam as atividades dentro do contexto hospitalar, embora ainda persistam disciplinas ditas básicas, como é o caso da Farmacologia. A disciplina é organizada em atividades didáticas, como aulas teóricas, painéis e discussões e exercícios em grupos com cerca de 20 a 25 alunos. Até o ano de 2001, as aulas teóricas eram ministradas para o conjunto dos alunos (aproximadamente 96 por semestre), passando a ser oferecidas em grupos menores a partir de então. As avaliações eram realizadas através de três provas e sete testes durante o período da disciplina (22 semanas), com média de oito horas de aulas semanais. A partir de 2001, o número de avaliações foi reduzido para duas provas e três testes.

A monitoria virtual é formada por um serviço de respostas a perguntas de alunos, que podem ser enviadas a qualquer hora do dia e são respondidas num prazo máximo de 48 horas pelos monitores, supervisionados pelos docentes; de um website (www.nesc.ufrj.br/epidemiologia), disponibilizado a partir do final do segundo semestre de 2001, onde estão disponíveis as FAQ (do inglês Frequently Asked Questions, coletânea de perguntas encaminhadas ao serviço de monitoria virtual, respondidas pela equipe do serviço), o material da disciplina (exercícios e textos de referência), links para páginas de Epidemiologia, Bioestatística, Saúde Pública e Medicina, entre outros; e de um serviço de mala-direta eletrônica onde são veiculadas mensagens sobre a disciplina de caráter administrativo (mudanças de aulas etc.) e dicas culturais (dicas sobre artigos recentemente publicados, por exemplo).

Este artigo avalia a utilização e a receptividade da monitoria virtual no período compreendido entre o primeiro semestre de 2000 e o primeiro semestre de 2002, buscando identificar possíveis padrões de uso do serviço, além de compreender melhor a relação dos alunos com o aprendizado de Epidemiologia e avaliar o uso da Internet como ferramenta complementar de ensino.

\section{Metodologia}

O estudo envolveu diferentes estratégias de avaliação, compreendendo a análise das mensagens enviadas e a avaliação, pelos alunos, da monitoria 
virtual (serviços de tutoria virtual e mala-direta) e do site.

Foram compiladas todas as mensagens enviadas para o serviço de monitoria virtual entre o primeiro semestre de 2000 e o primeiro semestre de 2002, destacando-se, em cada mensagem, a identificação do aluno, data e hora de envio, natureza da pergunta e quantidade de perguntas contidas em cada mensagem. Quanto à natureza, as perguntas foram classificadas como "dúvidas administrativas" (perguntas referentes ao funcionamento da disciplina de Epidemiologia), "dúvidas acadêmicas" (perguntas sobre conteúdos de Epidemiologia), "críticas ou sugestões" e "outros".

A partir do segundo semestre de 2000 foi aplicado, ao fim de cada semestre letivo, um questionário de avaliação do serviço de monitoria virtual. Esse questionário de autopreenchimento era anônimo e incluía perguntas objetivas relativas ao uso ativo (envio de mensagens) e passivo (recebimento de mensagens do serviço de mala-direta), satisfação com as respostas recebidas, importância atribuída ao serviço de monitoria virtual para o aprendizado de Epidemiologia e influência do serviço de monitoria virtual na freqüência de uso da Internet. O questionário continha também perguntas abertas avaliando fatores que dificultaram o uso do serviço e a opinião dos alunos sobre a extensão do serviço de monitoria virtual e outras disciplinas. Consideramos como tendo tido "acesso direto" ao serviço os alunos que receberam, em seus endereços eletrônicos, mensagens enviadas através do serviço de mala-direta. Tiveram "acesso indireto" os alunos que receberam as mensagens da monitoria virtual através de seus colegas.

No primeiro semestre de 2002 foi aplicado um questionário de avaliação do site, pelo qual foram avaliados, através de questões objetivas, o número de acessos de cada aluno ao longo do semestre letivo e o motivo que os levou a acessar o site pela primeira vez. Foram avaliados também a utilidade de cada uma das diversas seções do site, a adequação do conteúdo, apresentação do conteúdo, velocidade de acesso e tamanho dos arquivos disponíveis para download, além dos motivos que levaram alguns alunos a não terem acessado o site. O questionário envolvia ainda um espaço para sugestões e críticas ao site.

Na Tabela 1 é apresentada a distribuição de alunos correspondente a cada uma das avaliações realizadas. Foram construídas séries temporais do número de mensagens segundo horário e semana do curso. Os resultados relativos às categorias das mensagens enviadas e às questões abordadas nos questionários de avaliação do serviço de tutoria virtual e do site foram apresentadas como freqüências absolutas e relativas. Com relação à utiliza- 
ção, pelos alunos, dos diferentes serviços oferecidos (envio de mensagens; acesso direto ou indireto às mensagens enviadas através dos serviços de tutoria virtual; e mala-direta) e a importância das dúvidas acadêmicas no conjunto das perguntas enviadas, os resultados apresentados foram apresentados como proporções e os respectivos intervalos de confiança de 95\% calculados pelo método exato. A correlação entre a razão do número de perguntas por mensagens e a proporção de perguntas como dúvidas acadêmicas foi medida pelo coeficiente de correlação de Pearson. As análises foram realizadas utilizando os programas Epi-Info 6.04 (Dean et al., 1994) e Stata 7 (StataCorp, 2001).

Tabela 1 - Número de alunos matriculados e que responderam aos questionários de avaliação, por semestre letivo

\begin{tabular}{lccccccc}
\hline & $\begin{array}{c}\text { Total de alunos } \\
\text { matriculados }\end{array}$ & $\begin{array}{c}\text { Responderam à } \\
\text { avaliação da } \mathbf{M V}^{* * *}\end{array}$ & $\begin{array}{c}\text { Responderam à } \\
\text { avaliação do site*** }\end{array}$ \\
\hline $2000-1$ & $\mathbf{N}$ & $\mathbf{( \% )}$ & $\mathbf{N}$ & $\mathbf{( \% )}$ & $\mathbf{N}$ & $\mathbf{( \% )}$ \\
$2000-2$ & 101 & $100 \%$ & - & - & - & - \\
$2001-1$ & 96 & $100 \%$ & 88 & $91,7 \%$ & - & - \\
$2001-2$ & 99 & $100 \%$ & 82 & $82,8 \%$ & - & - \\
$2002-1$ & 93 & $100 \%$ & 79 & $95,2 \%$ & - & - \\
\hline Todos os semestres estudados & 479 & - & 325 & - & 77 & - \\
\hline
\end{tabular}

* Universo de alunos considerados nas análises em relação ao envio de mensagens.

** Universo de alunos considerados nas análises sobre o recebimento direto e indireto de mensagens

*** Universo de alunos considerados nas análise sobre o acesso ao site

\section{Resultados}

Durante o período analisado, a monitoria virtual recebeu 153 mensagens contendo 239 perguntas. A proporção de alunos matriculados que enviou mensagens para o serviço foi inferior a $20 \%$ nos diferentes semestres letivos, exceto para 2000/1 (Tabela 2). Com exceção do último semestre letivo, 
a proporção de alunos que relatou ter recebido ao menos uma mensagem diretamente em sua caixa postal foi superior a $60 \%$. Já o acesso indireto, i.e., alunos que tiveram contato com mensagens enviadas pela equipe do projeto através do contato com outro aluno, ficou em torno de $10 \%$ em todos os semestres (Tabela 2).

Tabela 2: Proporção de uso dos serviços de monitoria virtual segundo tipo e período semestre letivo*

\begin{tabular}{|c|c|c|c|c|c|c|}
\hline & \multicolumn{2}{|c|}{$\begin{array}{c}\text { Envio de } \\
\text { Mensagens }\end{array}$} & \multicolumn{2}{|c|}{$\begin{array}{l}\text { Acesso direto às } \\
\text { mensagens enviadas } \\
\text { através dos serviços de } \\
\text { tutoria e mala direta }\end{array}$} & \multicolumn{2}{|c|}{$\begin{array}{l}\text { Acesso indireto às } \\
\text { mensagens enviadas } \\
\text { através dos serviços de } \\
\text { tutoria e mala direta }\end{array}$} \\
\hline & $\%$ & IC $95 \%$ & $\%$ & IC $95 \%$ & $\%$ & IC $95 \%$ \\
\hline $2000-1$ & $26,7 \%$ & 18,4 a 36,4 & - & - & - & - \\
\hline $2000-2$ & $12,5 \%$ & 6,6 a 20,8 & $61,4 \%$ & 50,4 a 71,5 & $12,5 \%$ & 6,4 a 21,2 \\
\hline 2001-1 & $18,2 \%$ & 11,1 a 27,2 & $63,4 \%$ & 52,0 a 73,8 & $12,2 \%$ & 6,0 a 21,3 \\
\hline $2001-2$ & $19,3 \%$ & 11,9 a 28,8 & $73,4 \%$ & 73,4 a 82,7 & $8,8 \%$ & 3,6 a 17,4 \\
\hline $2002-1$ & $16,6 \%$ & 9,6 a 25,9 & $48,6 \%$ & 37,0 a 60,4 & $10,5 \%$ & 4,6 a 19,7 \\
\hline
\end{tabular}

* Os totais empregados para o cálculo das proporções são apresentados na tabela 1

Observou-se variação no total de mensagens e perguntas enviadas segundo o período estudado, havendo tendência ao encaminhamento de um maior número de perguntas por mensagens nos semestres em que foram enviadas mais mensagens. A análise da série semanal do número de mensagens enviadas mostra uma flutuação ao longo das semanas do curso, com maior concentração de mensagens enviadas perto das datas de provas da disciplina. A associação entre o envio de mensagens e as provas da disciplina é particularmente nítida em 2000/1 (Figura 1). Nesse período o calendário oficial do curso foi interrompido por uma greve, o que influenciou negativamente a dinâmica de envio de mensagens ao serviço, assim como o próprio andamento da disciplina. Considerando o conjunto das 153 mensagens, verificamos que $54(35,3 \%)$ foram enviadas nos quatro dias que antecediam as provas da disciplina. 
Ana Luiza Alfaya Gallego Soares et al.

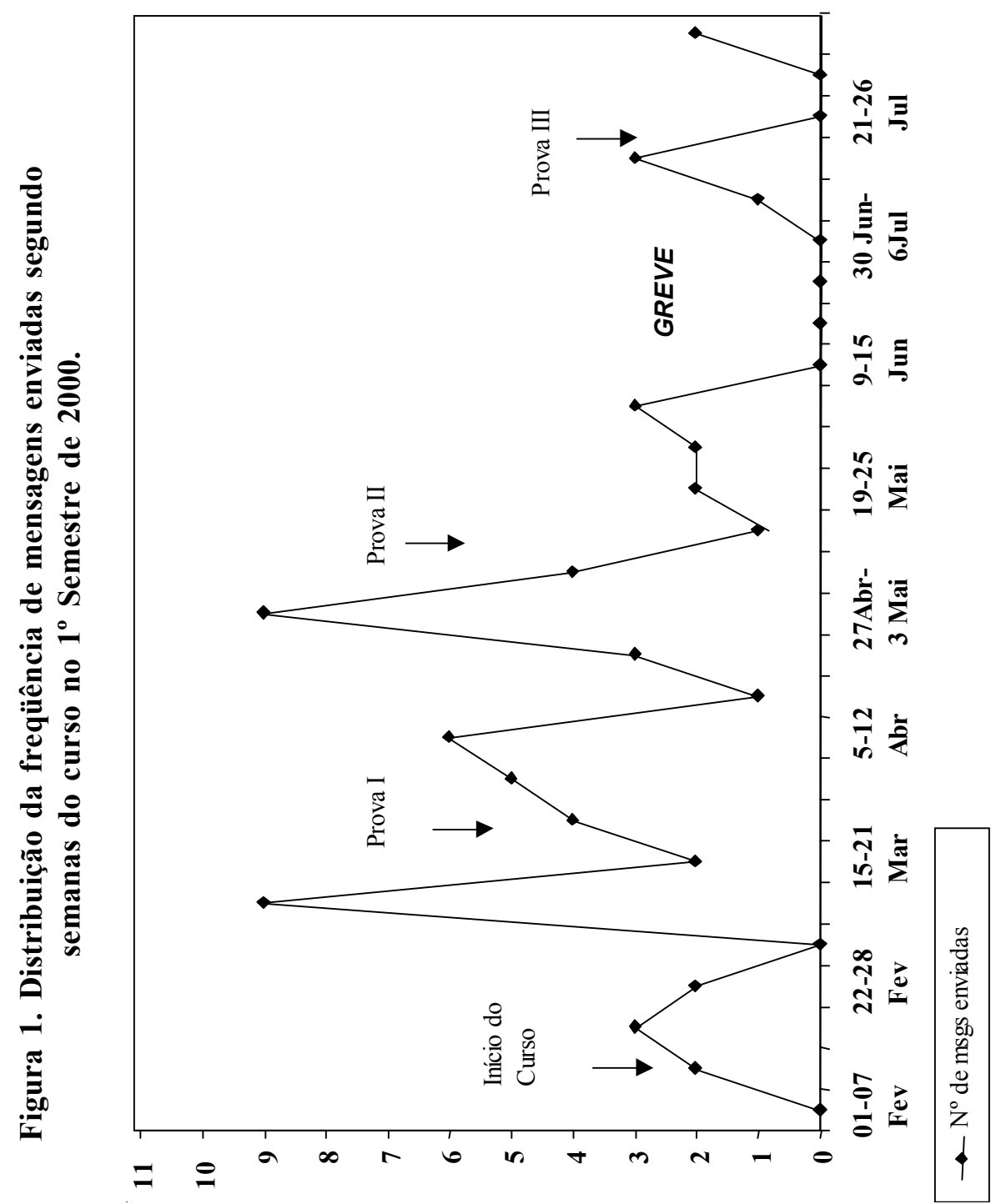


Quanto à distribuição das mensagens segundo horário do envio, a maioria $(\mathrm{n}=73 / 153 ; 47,7 \%)$ foi enviada entre 18 e $6 \mathrm{~h}$ (Figura 2). Com relação ao tipo de perguntas recebidas, 59,0\% $(\mathrm{n}=141 / 239)$ continham "dúvidas acadêmicas" e $27,6 \%(n=66 / 239)$ eram "dúvidas administrativas". A proporção de perguntas com dúvidas acadêmicas em relação ao total de perguntas enviadas variou nos diferentes períodos, sendo maior naqueles em que maior número de mensagens foi enviado (Figura 3). Também se observou correlação positiva entre a razão do número de perguntas enviadas por mensagem e a proporção de perguntas que continham "dúvidas acadêmicas" ( $\mathrm{r}=0,95$; IC $95 \% 0,46$ a 0,99$)$.

Figura 2. Distribuição da freqüência de mensagens enviadas segundo período do dia.

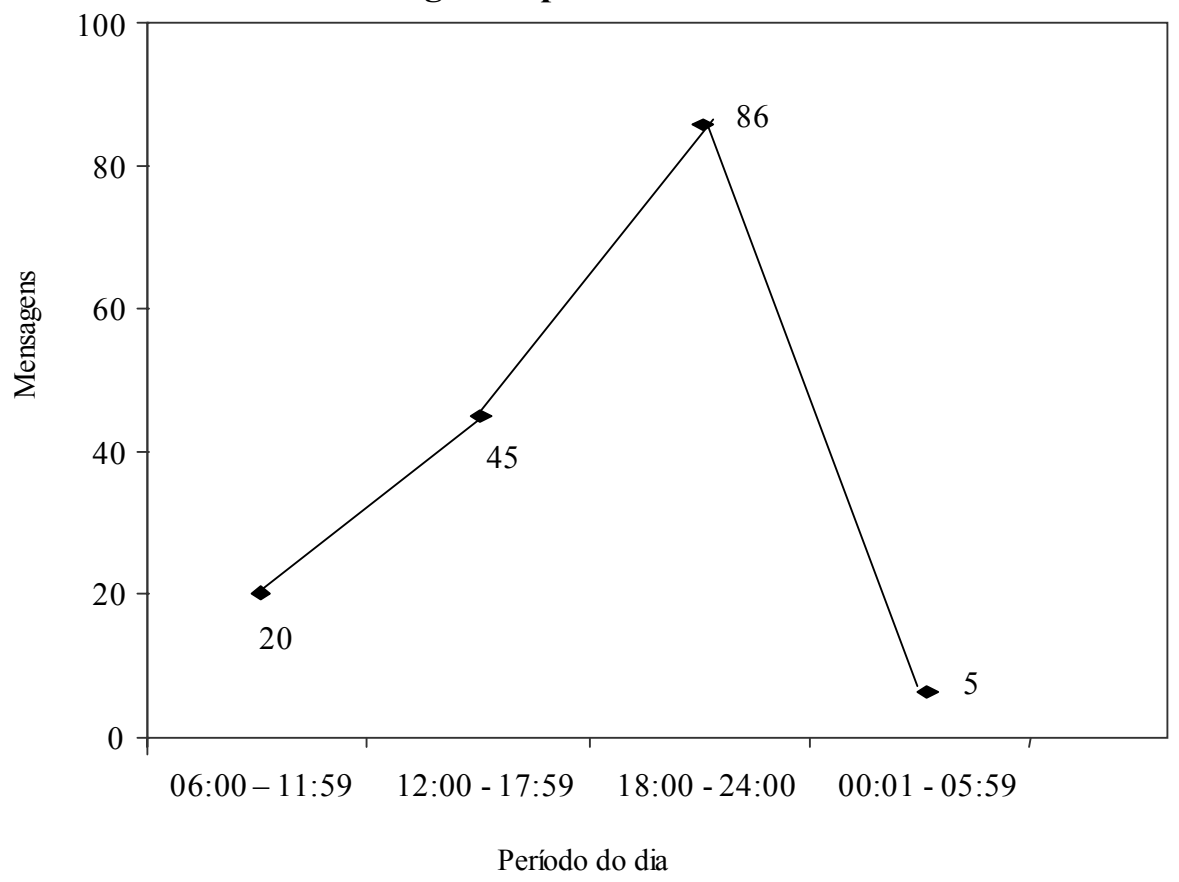


Ana Luiza Alfaya Gallego Soares et al.

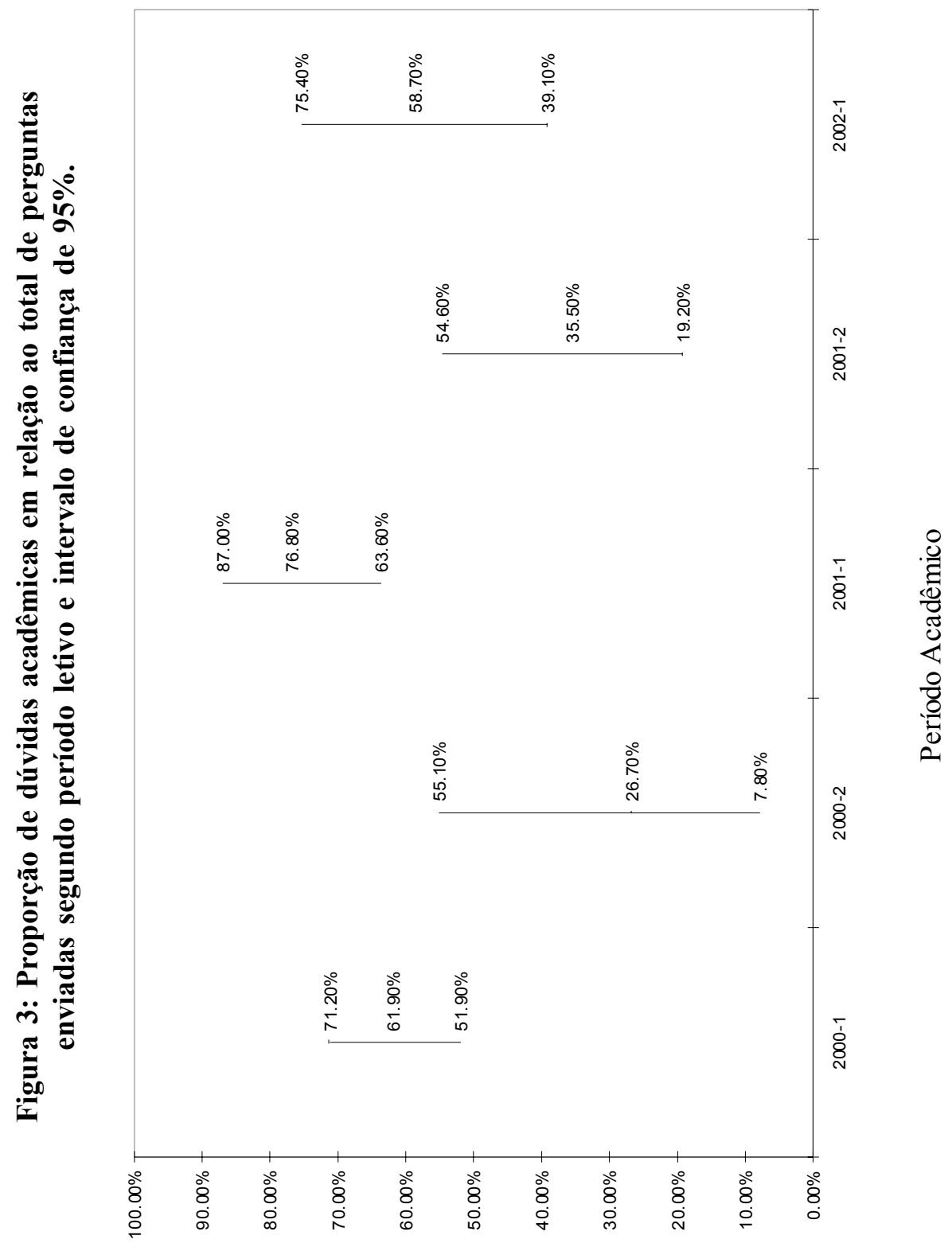

48 PHYSIS: Rev. Saúde Coletiva, Rio de Janeiro, 13(1):39-58, 2003 
O questionário de avaliação da monitoria virtual foi preenchido por $86,0 \%$ $(n=325 / 378)$ dos alunos matriculados na disciplina a partir do segundo semestre de 2000 (ver Tabela 1), dos quais 15,1\% ( $n=49 / 325)$ referiram ter escrito para a monitoria virtual; destes, $60,7 \%(\mathrm{n}=30 / 325)$ ficaram totalmente satisfeitos com a(s) resposta(s) recebida(s), 14,3\% $(\mathrm{n}=7 / 325)$ ficaram parcialmente satisfeitos e $24,5 \%(n=12 / 325)$ referiram não ter recebido resposta. Dos 325 alunos que responderam ao questionário de avaliação, 164 responderam a uma pergunta sobre os motivos que os levaram a não usar o serviço de $\mathrm{MV}$, quando fosse o caso. Os motivos mais citados foram não ter julgado necessário enviar mensagens ou ter solucionado todas as dúvidas em sala de aula (Tabela 3). Em todos os semestres estudados, apenas 17,8\% $(n=58 / 325)$ dos alunos afirmaram que a monitoria virtual aumentou sua freqüência de uso da Internet, enquanto $87,7 \%$ ( $\mathrm{n}=285 / 325)$ consideram uma boa idéia que outras disciplinas utilizem serviços de monitoria virtual.

Tabela 3. Motivos que levaram o aluno a não enviar mensagens para a Monitoria Virtual (total de alunos que responderam $=164$ )

\begin{tabular}{lll}
\hline Motivos & N & $\%$ \\
\hline Não acharam necessário & 54 & $32,9 \%$ \\
Solucionaram dúvidas em sala de aula & 48 & $29,3 \%$ \\
Não têm acesso a e-mail/Internet & 36 & $21,9 \%$ \\
Não tiveram tempo & 20 & $12,2 \%$ \\
Sem interesse pela disciplina & 6 & $3,7 \%$ \\
\hline
\end{tabular}

A partir de 2001/2 foi incluída no questionário pergunta sobre a utilidade dos diversos serviços oferecidos pela monitoria virtual. A possibilidade de ter acesso aos materiais da disciplina foi o serviço da monitoria virtual mais citado como útil pelos alunos $(\mathrm{n}=112 / 155 ; 72,3 \%)$, seguido pelo envio de informes sobre a disciplina $(\mathrm{n}=88 / 155 ; 60,6 \%)$, ficando o serviço de tiradúvidas em terceiro lugar, o qual foi considerado útil por metade dos alunos $(\mathrm{n}=78 / 155 ; 50,3 \%)$ (Tabela 4). 
Tabela 4. Proporção de alunos que consideram úteis os serviços da Monitoria Virtual segundo tipo do serviço oferecido (Número de alunos $=155$ )

\begin{tabular}{lrc}
\hline & N & $\%$ * \\
\hline Acesso a materiais do curso & 112 & $72,3 \%$ \\
Envio de informes da disciplina & 94 & $60,6 \%$ \\
Tira-dúvidas por e-mail & 78 & $50,3 \%$ \\
Dicas Culturais & 27 & $17,4 \%$ \\
Nenhum & 11 & $3,4 \%$ \\
Outros & 2 & $1,7 \%$ \\
\hline
\end{tabular}

* A soma excede $100 \%$, pois cada aluno pode assinalar mais de um serviço como útil

O questionário de avaliação do site foi preenchido por $85,6 \%(\mathrm{n}=77 / 90)$ dos alunos matriculados no primeiro semestre de 2002 e, destes, 49 (63,6\%; IC $95 \% 51,9 \%$ a $74,3 \%$ ) acessaram o site (ver Tabela 1). Entre os que o fizeram, a maioria $(n=32 / 49 ; 65,3 \%)$ teve mais que 2 e menos que 10 acessos; $18,4 \%(n=9 / 49)$ tiveram apenas um acesso e $16,3 \%(n=8 / 49)$ acessaram o site mais de 10 vezes durante o semestre. Em primeiro lugar, a grande maioria dos alunos ( $\mathrm{n}=46 / 49 ; 93,9 \%)$ buscava obter material didático da disciplina. A seção "sobre o curso", onde foi disponibilizado o material da disciplina, foi a mais citada como útil/interessante por $87,8 \%$ dos alunos que acessaram o site ao menos uma vez $(n=43 / 49)$.

A maioria dos alunos classificou a velocidade de acesso ao site como "regular" ou "boa" ( $\mathrm{n}=38 / 49 ; 77,6 \%)$; o tamanho dos arquivos disponíveis para download como "bom" ( $\mathrm{n}=34 / 49 ; 70,8 \%)$; o conteúdo do site como "bom" (n=38/49; 77,6\%) e a apresentação desse conteúdo como "bom" ou "excelente" ( $\mathrm{n}=37 / 49 ; 75,5 \%)$.

Entre os alunos que não acessaram o site, a maioria $(\mathrm{n}=8 / 13,60,7 \%)$ não o fez por falta de interesse em Epidemiologia e 28,6\% $(n=4 / 13)$ por falta de interesse pela Internet. Houve poucas críticas e sugestões para melhoria do site, sendo a quase totalidade referente ao aumento da quantidade de material didático da disciplina disponível e/ou à disponibilidade de gabaritos e notas de provas. 


\section{Discussão}

A monitoria virtual, citada como exemplo de usos da informática dentro do ensino da Epidemiologia pelo III Plano Diretor para o Desenvolvimento da Epidemiologia no Brasil (Abrasco, 2000), enquadra-se em três das seis proposições de formas de inserção das tecnologias da informação dentro da educação médica, formuladas pela Federação Mundial de Educação Médica (WFME, 1998): a) espaço para perguntas e respostas relacionadas ao conteúdo de palestras e do curso; b) provisão de boletins com informações sobre o curso; c) recepção de comentários para revisão do curso. As demais proposições são: d) grupos para discussão entre estudante e staff; e) provisão de material para auto-avaliação; f) meio de assegurar a entrega e retorno de trabalhos dos estudantes.

É possível que o elemento mais inovador presente nessa iniciativa seja a perspectiva de ampliação das possibilidades de diálogo entre alunos e professores. Quando analisamos elementos do discurso diário a respeito do ensino médico, fica claro que sua visão tradicional se inscreve no modelo de educação bancária (Freire, 1967; Freire, 1970; Swanson et al., 1993), no qual o conhecimento deve ser transmitido, comunicado unidirecionalmente aos alunos, que são aqueles que "não sabem", em oposição aos professores, que são aqueles que "sabem". Por isso a ênfase da monitoria dos cursos sobre atividades do tipo palestra, por isso a relutância e a dificuldade que professores referem para lidar com grupos menores de estudantes dentro da metodologia de aprendizado baseado em problemas (ABP ou PBL), ou com conteúdos fora da sua área de "domínio" (Swanson et al., 1993). De alguma forma, o grande volume, a velocidade de produção e de acesso ao conhecimento médico vêm desconstruindo os degraus - ou seriam muros? - que separavam os alunos dos professores, pois uma vez que abarcar todo o conhecimento torna-se inviável, todos passam à categoria de aprendizes da medicina - uns com mais, outros com menos tempo de estrada, porém aprendizes. Abre-se a porta para a vivência de um dos pontos de partida das reflexões e propostas de Paulo Freire (Freire, 1967, 1970 e 1997) sobre educação, como o da "incompletude humana", que abre a todos a possibilidade de aprendizado e crescimento, nos faz humildes e nos conduz ao próximo.

Ao se criar a MV com disponibilidade permanente ao diálogo, intermediada pela figura dos monitores, i.e. alunos "com um pouco mais de estrada", é fortalecida a possibilidade de comunicação horizontal com os alunos, não 
mais para os alunos. Os mais tímidos e reservados em sala de aula têm à disposição um espaço onde se expressar (Owston, 1997). Facilita-se o debate a respeito de questionamentos que surjam em momentos posteriores de reflexão dos alunos, muito após o término das palestras. Dito isto, desejamos assinalar que a proposta da MV não foi, em momento algum, de substituição do ensino presencial como uma forma de educação à distância, mas uma complementação do mesmo que multiplicasse as oportunidades de interação com o alunado.

Todavia, nem tudo anda sempre conforme desejamos, nem todas as possibilidades são efetivamente aproveitadas... O diálogo não substitui facilmente o não-diálogo (Freire, 1967). Embora 67\% dos alunos tenham recebido, direta ou indiretamente, nossas mensagens, o envio de mensagens para o serviço de tutoria não ultrapassou $26,9 \%$ do total dos alunos inscritos, mesmo nos semestres mais "ativos". Adicionalmente, verificamos a concentração do envio das mensagens nas vésperas das provas e maior valorização, por parte dos alunos, dos serviços que envolviam disponibilização do material e informes sobre a disciplina. Assim, os serviços da MV que eram, para nós, meros auxiliares, se tornaram centrais para os alunos. Em vez de contribuir para aumentar a reflexão sobre os conteúdos e melhorar a compreensão dos temas, a MV foi primordialmente utilizada como um recurso para melhorar o desempenho nas avaliações da disciplina.

O objetivo principal deste trabalho foi avaliar o processo de utilização de uma nova intervenção educacional. Sendo assim, a metodologia empregada permitiu apenas realizar uma análise exploratória dos possíveis motivos associados a barreiras na incorporação da intervenção proposta. Reconhecendo essas limitações, acreditamos, entretanto, que algumas hipóteses possam ser levantadas, as quais futuramente serão mais bem avaliadas, através de entrevistas mais detalhadas com alunos e professores do curso de medicina.

Em primeiro lugar, existe a dificuldade de incorporação de uma nova cultura de aprendizagem (Greenhalgh, 2001). Peixoto et al. (2002), realizando inquérito sobre fontes de estudo utilizadas por alunos de medicina da Região Sudeste, incluindo alunos da UFRJ, verificaram que os mesmos utilizavam recursos mais tradicionais, como livros, notas de aula e apostilas. Como parte do projeto de monitoria virtual, os alunos respondem a um questionário para avaliação do acesso e do padrão de utilização de computadores e da Internet ${ }^{7}$. A análise dos dados coletados até o momento mostra que mais de $90 \%$ dos alunos possuem computador, e-mail e acesso à Internet, mas que menos de $30 \%$ fazem uso diário desta. Outro resultado do inquérito 
que chama a atenção é o fato de poucas disciplinas ao longo do ciclo básico terem demandado uso da Internet. Por outro lado, a falta de intimidade com o meio eletrônico foi motivo alegado por mais de $25 \%$ dos alunos na presente análise como razão de não terem utilizado os serviços oferecidos.

Ainda com relação ao estilo de aprendizado, existe o pragmatismo acentuado dos estudantes de medicina em relação à obtenção de notas para provas, enquanto objetivo principal na disciplina. Nossos achados corroboram outro resultado obtido por Peixoto et al. (2002). Os autores observaram que o principal objetivo referido pelos alunos entrevistados para a compra de um livro-texto era sua adequação para o estudo dirigido às provas curriculares. Por fim, a falta de laboratórios de informática em número suficiente e a grade curricular sobrecarregada do curso de medicina representam barreiras adicionais para utilização mais freqüente da Internet e de intervenções educacionais nela baseadas. Cabe ressaltar que o pico do envio de mensagens ocorreu nos horários em que os alunos se encontravam fora da universidade. Esses resultados foram confirmados por estudo realizado a partir da análise do banco de dados dos acessos ao site (análise de $l o g$ ), sendo verificado predomínio dos acessos no período compreendido entre 18 e $24 \mathrm{~h}$ e um baixo acesso realizado a partir de máquinas com endereço IP (Internet Protocol) do domínio da UFRJ (Coeli et al., 2002).

Em segundo lugar, a inserção da monitoria virtual na disciplina de Epidemiologia não se deu de forma completa, exceto no que diz respeito ao fornecimento do material do curso. A incorporação de tecnologias de aprendizado assistido por computador em cursos tradicionais não se faz de forma imediata, sendo necessário garantir o envolvimento dos professores, bem como os recursos necessários para aquisição de hardware, software e a capacitação de pessoal (Greenhalgh, 2001). Apenas dois professores que ministram a disciplina de Epidemiologia fazem parte da equipe responsável pelo projeto de monitoria virtual. Também enfrentamos dificuldades de infraestrutura universitária: o acesso ao site foi por vezes inviabilizado por motivos técnicos, desacreditando o serviço junto ao corpo discente. Tais dificuldades técnicas podem explicar a proporção significativa $(24,5 \%)$ de alunos que referiram não ter recebido respostas às suas mensagens - as quais nunca chegaram até nós - e os $2,2 \%$ de alunos queixando-se de atraso excessivo na resposta - situação que ocorreu, apesar dos nossos esforços, porém em número menor de casos, e que pode ter sido maximizada por atrasos em nosso servidor de e-mail. Não se pode deixar de citar a obrigatoriedade de a equipe estar sempre disponível, no sentido de verificar 
constantemente as contas de e-mail e produzir resposta aos questionamentos em tempo hábil. As mensagens enviadas nas vésperas das provas continham predominantemente mais de uma dúvida acadêmica. Um dos resultados observados em nosso estudo foi a elaboração positiva entre o número de perguntas enviadas por mensagem e a proporção de perguntas que continham dúvidas acadêmicas.

Por fim, a Epidemiologia e a Saúde Coletiva tendem a ser subvalorizadas e vistas como matérias periféricas por parte significativa dos estudantes de medicina (Stone, 1999; GMC, 2002). O desinteresse pela disciplina foi um dos motivos alegados pelos alunos para o não-envio de mensagens ou acesso ao site. Parte disso decorre da segregação $\operatorname{artificial}^{8}$ que se estabelece, ainda que combatida nos textos curriculares e consensos de sociedade (GMC, 2002), entre os conteúdos ditos clínicos - os quais, para os estudantes, correspondem à grande aspiração de seu aprendizado - e aqueles cuja base repousa na Epidemiologia e na Saúde Coletiva - como se a base epidemiológica não estivesse intimamente relacionada com a clínica, por trás de todo processo de decisão diagnóstica, terapêutica e preventiva.

Esta separação artificial é reforçada pela própria inexperiência clínica dos estudantes no momento da educação em Epidemiologia, que não lhes proporciona visão suficientemente ampla para perceber a interdependência da disciplina no saber médico, pela própria estruturação dos cursos dentro das escolas médicas e pelo desconforto, de parte do corpo docente, em lidar e integrar tais elementos.

Cremos ser importante não perder de vista esse contexto, tão comum a um número tão grande de escolas médicas, se não à maioria delas, a fim de melhor compreender as dificuldades que circundam o ensino-aprendizagem epidemiológico. As dificuldades impostas pela segmentação artificial do saber médico (Camargo Jr., 1997) e pela falta de perspectiva do alunado quanto à integração clínico-epidemiológica, a qual de resto não se dá nos demais espaços de suas experiências de graduação, formam obstáculos significativos ao pleno sucesso da proposta.

Frente a tantas barreiras e obstáculos, acreditamos que o relato da experiência do Projeto de Monitoria Virtual possa auxiliar na reflexão sobre o processo de ensino-aprendizagem da Epidemiologia e da Saúde Pública nos cursos de medicina. Nossa expectativa de contribuição para maior reflexão do conteúdo estudado, ligada a uma atitude ativa de busca do conhecimento, só seria alcançada através de uma reformulação estrutural, não apenas da disciplina de Epidemiologia como também do currículo médico, formador de 
posturas de aprendizado dos alunos. Apesar disso, nossos resultados apontam para a necessidade de reformulações na proposta inicial, buscando, por um lado, melhor integração do projeto de Epidemiologia e, por outro, a ampliação da população-alvo inicial, visando a atingir alunos de outros períodos e professores de outras disciplinas.

Com relação ao último aspecto, a valorização do ensino de Saúde Pública no curso médico vem sendo apontada como fundamental para a melhoria da qualidade da formação profissional (GMC, 2002; Nickens, 1999; Stone, 1999). A estratégia recomendada para alcançar esse objetivo é a integração do ensino de Saúde Pública ao ensino das disciplinas clínicas, embora iniciativas neste sentido nem sempre sejam bem-sucedidas (Stone, 1999). O espaço virtual pode representar uma alternativa interessante na busca dessa integração. Nesse sentido, um dos objetivos da nova fase de nosso projeto é o desenvolvimento de um aplicativo educativo, em que casos clínicos serviriam de base para a reflexão sobre aspectos clínicos e epidemiológicos e a relação com as questões de saúde pública relevantes em nosso meio.

\section{Referências Bibliográficas}

ABRASCO. III Plano Diretor para o Desenvolvimento da Epidemiologia no Brasil, 2000 - 2004. p. 18-20.

BLUE, A. V. et al. Evaluation Methods for Prevention Education. Academic Medicine, v. 75, n. 7, jul. 2000.

BOELEN, C. et al. Defining and Measuring the Social Accountability of Medical Schools. World Health Organization. Disponível em: <http:// whqlibdoc.who.int/hq/1995/WHO_HRH_95.7.pdf> Acesso em: 24/02/2003. CAMARGO Jr., K. R. A Biomedicina. Physis: Rev. Saúde Coletiva, Rio de Janeiro, v. 7, n. 1, p. 45-68, 1997.

CARLILE, S. et al. Healthcare and the information age: implications for medical education. CMJA, v. 168 p. 340-343, 1998.

COELI, C. M. et al. A construção e utilização de um site voltado para o ensino de Epidemiologia para alunos do curso médico. Revista Brasileira de Educação Médica, Rio de Janeiro, v. 26, p. 66-66, 2002. Suplemento 1. DEAN, A. G. et al. Epi Info, Version 6: a word processing, database, and statistics program for epidemiology on micro-computers. Atlanta: Centers for Disease Control, 1994. 
FREIRE, P. Educação como Prática da Liberdade. 25. ed. Rio de Janeiro: Paz e Terra, 1967. 158p.

FREIRE, P. Pedagogia do Oprimido. 31. ed. Rio de Janeiro: Paz e Terra. 1970. 384p.

FREIRE, P. Pedagogia da Autonomia: saberes necessários à prática educativa. 1.ed. Rio de Janeiro, Paz e Terra: 1997. 185p.

GENERAL MEDICAL COUNCIL. Tomorrow's doctors: recommendations on undergraduate medical education. Londres, Jul. 2002. Disponível em: http://www.gmc-uk.org/med_ed/. Acesso em: 24/02/2003.

GREENHALGH, T. Computer assisted learning in undergraduate medical education. BMJ, v. 322 p. 40-44, 2001.

HAGDRUP, N. A. et al. Why? What? And How? IT provision for medical students in general practice. Medical Education, v. 33, p. 537-541, 1999. KOSCHMANN, T. Medical Education and computer literacy: learning about, through, and with computers. Academic Medicine, v. 70, n. 9, p. 818-821, set. 1995.

MacKENZIE, J. Grazing the Net - Raising a generation of free-range students. Phi Delta Kapan, p. 26-31, Set. 1998.

NICKENS, H. It's about time - the medicine/public health initiative. Am J Prev Med, v. 16, p. 20-21, 1999. Suppl. 3.

OWSTON, R. D. The World Wide Web: a Technology to enhance Teaching and Learning? Educational Researcher, v. 26, n. 2, p. 27-33, Mar. 1997. PEIXOTO, M. A. P. et al. Fontes de Estudo na Escola Médica - Recursos Instrucionais Utilizados por Alunos de Medicina da Região Sudeste do Brasil. Revista Brasileira de Educação Médica, Rio de Janeiro, v. 24, n. 3, out./dez. 2000.

RAPPLEYE, W.C. Medical Education: final report of the Comission on Medical Education. New York: 2002.

RIBEIRO, V. M. B. Condições da Formação do Profissional na Área da Saúde na UFRJ: a Faculdade de Medicina. Revista Brasileira de Educação Médica, Rio de Janeiro, v. 26, n. 2, p. 115-124, mai./ago. 2002.

STATACORP. Stata Statistical Software: Release 7.0. College Station, TX: Stata Corporation. 2001.

STONE, D. H. Public health in the undergraduate medical curriculum - can we achieve integration? Journal of Evaluation in Clinical Practice, v.6, n. 1, p. 9-14, Out. 1999.

SWANSON, A. G. et al. ACME-TRI Report: Educating Medical Students, Assessing change in Medical Education - The Road to Implementation. 
Academic Medicine, v. 68, n. 6, suppl. Jun., p. S7-S46, 1993.

WORLD FEDERATION FOR MEDICAL EDUCATION. Guidelines for using Computers in Medical Education. Medical Education, v. 32, p. 205208, 1998.

WILKES, M. B., J. Evaluating educational interventions. $B M J$, v. 318, p. 1269-1272, Mai. 1999.

\section{NOTAS}

${ }^{1}$ Aluna de graduação, Faculdade de Medicina, Universidade Federal do Rio de Janeiro.

2 Aluna de graduação, Faculdade de Medicina, Universidade Federal do Rio de Janeiro.

${ }^{3}$ Médico residente em Saúde da Família pela Faculdade de Medicina de Marília (FAMEMA).

4 Professora Adjunta do Departamento de Medicina Preventiva, Faculdade de Medicina e Núcleo de Estudos de Saúde Coletiva, Universidade Federal do Rio de Janeiro.

5 Médica; pesquisadora do Núcleo de Estudos de Saúde Coletiva, Universidade Federal do Rio de Janeiro. Doutora em Ciências pela Faculdade de Medicina da Universidade de São Paulo (USP).

${ }^{6}$ Professor Adjunto do Instituto de Medicina Social, Universidade do Estado do Rio de Janeiro.

7 Os resultados relativos às duas primeiras turmas avaliadas são apresentados no artigo "Virtual Tutoring Project: evaluating the feasibility of using the Internet as an auxiliary medium to teach Epidemiology to undergraduate medical students", que será publicado no volume XX dos Cadernos de Saúde Coletiva.

${ }^{8}$ Esta segregação de conteúdos a princípio indissociáveis não se dá por acaso, mas reflete o caráter analítico fundador dentro da própria biomedicina, que se estende desde a separação de sistemas orgânicos, de resto integrados num mesmo organismo (Boelen et al., 1995). 


\section{ABSTRACT}

Utilization of a Virtual Tutoring Service for Teaching Epidemiology in Undergraduate Medical Education

This article discusses the teaching of course contents in Collective Health during undergraduate medical school, based on the experience at the School of Medicine of the Federal University in Rio de Janeiro (UFRJ), Brazil. Virtual Tutoring (VT) is used as a complementary teaching tool, comprising a service that provides answers to students' questions through a website and an e-mail list. This article discusses the results from the evaluation of the utilization of (and receptiveness to) VT services from 2000 to 2002. The methodology consisted of an analysis of messages submitted and student evaluation of the services. During this period, VT received 153 messages containing 239 questions. More than $60 \%$ of the students had received some message in their mailboxes, and $10 \%$ had contacted the messages through other students. Fewer than $20 \%$ had sent messages to the VT. The analysis of the number of messages submitted showed a variation during the course schedule, with a peak concentration of questions close to exam time (35\%). The messages contained "academic doubts" in $59 \%$ of the cases. The VT was evaluated by $86 \%$ of the students, of whom $61 \%$ were completely satisfied with the answers they had received, some $18 \%$ reported that VT had increased their frequency of Internet use, and $88 \%$ agreed with the idea of incorporating the service into other disciplines. The website was accessed by $64 \%$ of the students. The majority (65\%) had made from 3 to 10 hits. The study methodology allowed for an exploratory analysis of possible factors associated with the assimilation of the proposed intervention.

Keywords: Epidemiology; medical education; undergraduate studies; computer-mediated communications; Internet.

Recebido em: 29/04/2003.

Aprovado em: 08/05/2003. 